\title{
Optimum Maneuvers of a Skip Vehicle with Bounded Lift Constraints ${ }^{1}$
}

\author{
A. Busemann ${ }^{2}$, N. X. Vinh ${ }^{3}$, and G. F. Kelley ${ }^{4}$ \\ Communicated by T. N. Edelbaum
}

\begin{abstract}
This paper presents the analytical solutions of the problem of optimum maneuvering of a glide vehicle flying in the hypervelocity regime. The investigation is based on the approximation of Allen and Eggers; namely, that along the fundamental part of a reentry or ascent trajectory, the aerodynamic forces greatly exceed the components of the gravitational force in the directions tangent and normal to the flight path.

The problem consists of finding an optimal control law for the lift such that the final velocity or the final altitude is maximized. This problem can be viewed as bringing the vehicle to the best condition for interception, penetration, or making an evasive maneuver.

If the range is free, the optimal lift control is obtained in closed form. If the lift control is bounded, then bounded control is optimal whenever it is reached. The switching sequences for different cases are discussed, and it is shown that there are at most two switchings. Bounded lift control is always at the ends of the optimal trajectory; for the case of two switchings, the optimal trajectory has an inflection point.
\end{abstract}

\section{Introduction}

In recent years, much attention has been focused on the problem of the optimum maneuvering of a glide vehicle. However, few analytical results

${ }^{1}$ Paper received April 2, 1968; in revised form, August 30, 1968. The authors wish to thank the National Aeronautics and Space Administration for the Grant No. NGR-06-003-033 under which this work was carried out.

${ }^{2}$ Professor, Department of Aerospace Engineering Sciences, University of Colorado, Boulder, Colorado.

${ }^{3}$ Associate Professor, Department of Aerospace Engineering, University of Michigan, Ann Arbor, Michigan.

${ }^{4}$ Captain, USAF, and Instructor, Department of Astronautics and Computer Sciences, USAF Academy, Colorado Springs, Colorado. 
have been obtained; most of the work has concentrated on numerical analysis. In the light of the excellent work of Contensou (Ref. 1), it appears that, if the acceleration of gravity is neglected (that is, if the approximation of Allen and Eggers is used, Refs. 2-3), a detailed analytical study of the problem can be achieved. Such analytical solutions may be less accurate than numerical solutions, but they are useful in many respects. They show the main characteristics of the optimal control law and permit a rapid comparative analysis of different trajectories. Also, in the region of the state space where the approximations are justified, analytical solutions give an adequate answer to the problem.

In this paper, we consider the motion of a vehicle flying in the hypervelocity regime in a vertical plane with engine shut off at all points of the flight path. The trajectory can be controlled by an elevator, thus varying the aerodynamic forces acting on the flying object. The system has one independent variable, the time $t$, and four dependent variables, namely, the horizontal distance $x$, the altitude $z$, the velocity $V$, and the path angle $\gamma$ (Fig. 1).

If we assume a drag function of the form $D=D(z, V, L)$ and if the lift program $L=L(t)$ is prescribed at all times, then, for a given initial condition, the trajectory of the vehicle is uniquely determined. The problem consists of finding an optimal control law for the lift and drag forces to bring the vehicle from a known initial condition to a terminal condition such that a certain flight element (the altitude or the velocity) is maximized. This problem can be viewed as bringing the vehicle to the best condition for interception, penetration, or making an evasive maneuver.

Since the analysis neglects the gravitational force, it applies to the fundamental part of a reentry or ascent trajectory; this is the part in which,

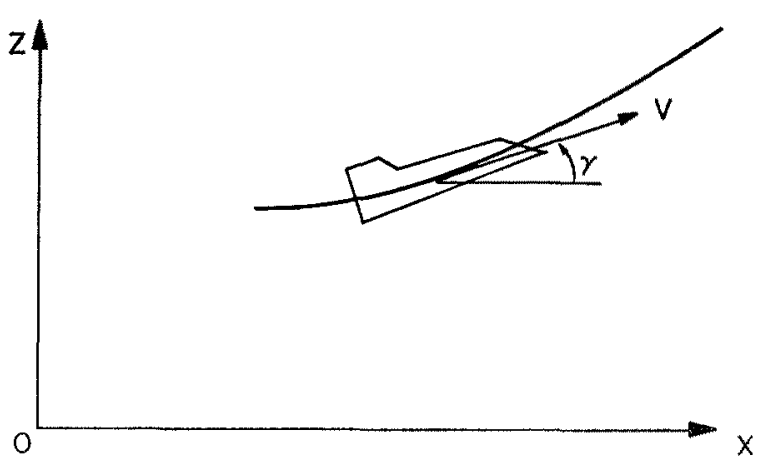

Fig. 1. Geometry of the trajectory. 
on the average, the aerodynamic forces greatly exceed the components of the gravitational force in the directions tangent and normal to the flight path. The flight path involves a relatively short range and altitude and, hence, can be investigated within the framework of the nonrotating, flat-Earth model.

\section{Formulation of the Problem}

To define a control parameter, we assume for the vehicle a generalized drag polar of the form

$$
C_{D}=C_{D 0}+K C_{L}{ }^{n}, \quad n>1
$$

where the zero-lift coefficient $C_{D 0}$, the induced drag factor $K$, and the exponent $n$ are assumed independent of the Mach number and the Reynolds number for the velocity-altitude range of the maneuver. For thin-winged configurations operating in the hypervelocity domain, $n$ is close to $3 / 2$.

We define a control parameter $\lambda$ by the relation

$$
\lambda^{n}=\left[(n-1) K / C_{D 0}\right] C_{L}^{n}
$$

Then, for each value of $\lambda$, the lift and drag coefficients are given by

$$
C_{L}=C_{L}^{*} \lambda, \quad C_{D}=\left(C_{D}^{*} / n\right)\left[n-1+\lambda^{n}\right]
$$

where $C_{L}^{*}$ and $C_{D}^{*}$ are the lift and drag coefficients corresponding to the maximum lift-to-drag ratio $E^{*}$, that is,

$$
\begin{aligned}
C_{L}^{*} & =\left[C_{D 0} /(n-1) K\right]^{1 / n}, \quad C_{D}^{*}=[n /(n-1)] C_{D 0}, \\
E^{*} & =(1 / n)\left[(n-1) / C_{D 0}\right]^{1-1 / n}(1 / K)^{1 / n}
\end{aligned}
$$

We note that $\lambda=1$ corresponds to maximum lift-to-drag ratio. We may assume that the control space is bounded, that is,

$$
|\lambda| \leqslant \lambda_{\max }
$$

The motion is governed by the equations (Ref. 4)

$$
\begin{aligned}
d x / d t & =V \cos \gamma \\
d z / d t & =V \sin \gamma \\
d \gamma / d t & =g L / V W \\
d V / d t & =-g D / W
\end{aligned}
$$


where $g$ is the acceleration due to gravity and $W$ is the weight of the vehicle. If the time is eliminated and the path angle is chosen as the new independent variable, we have the state equations

$$
\begin{aligned}
& d \xi / d \gamma=\cos \gamma / \omega \lambda \\
& d \omega / d \gamma=-\sin \gamma / \lambda \\
& d u / d \gamma=-\left[n-1+\lambda^{n}\right] / \lambda
\end{aligned}
$$

where the following dimensionless variables have been introduced

$$
\begin{aligned}
& \xi=x / \beta \\
& \eta=z / \beta, \quad \omega=g \beta C_{L}^{*} \rho S / 2 W=\left(\rho_{0} g \beta C_{L}^{*} S / 2 W\right) \exp (-\eta) \\
& u=\log [V / \sqrt{ }(\beta g)]^{n E^{*}}
\end{aligned}
$$

In Eq. (13), $S$ is a reference area and $\rho$ is the atmospheric mass density. The constants $\beta$ and $\rho_{0}$ are chosen so that the best average fit is obtained for the exponential approximation of the density variation in the altitude range of the maneuver. The initial conditions are

$$
\gamma=\gamma_{0}, \quad \xi=\xi_{0}=0, \quad \omega=\omega_{0}, \quad u=u_{0}
$$

and the final conditions are

$$
\gamma=\gamma_{1}, \quad \xi=\xi_{1}, \quad \omega=\omega_{1}, \quad u=u_{1}
$$

It is desired to find an optimal control $\lambda_{\text {opt }}$ to maximize a functional of the form

$$
J=C_{1} \xi_{1}+C_{2} \omega_{1}+C_{3} u_{1}
$$

For this purpose, we form the Hamiltonian

$$
H=p_{1} \cos \gamma / \omega \lambda-p_{2} \sin \gamma / \lambda-p_{3}\left[n-1+\lambda^{n}\right] / \lambda
$$

where the adjoint vector $\left(p_{1}, p_{2}, p_{3}\right)$ is defined by the adjoint equations

$$
\begin{aligned}
& d p_{1} / d \gamma=-\partial H / \partial \xi=0 \\
& d p_{2} / d \gamma=-\partial H / \partial \omega=p_{1} \cos \gamma / \omega^{2} \lambda \\
& d p_{3} / d \gamma=-\partial H / \partial u=0
\end{aligned}
$$

with the additional end conditions

$$
p_{i}\left(\gamma_{1}\right)=-C_{i}, \quad i=1,2,3
$$


The solution is obtained by integrating the systems of state equations (10)-(12) and adjoint equations (18)-(20) using the end conditions (14), (15), (21) and a control $\lambda=\lambda_{\text {opt }}$ such that, at each instant, the Hamiltonian $H$ takes its smallest value.

First, from the adjoint equations we have the first integrals

$$
p_{1}=\text { Const, } \quad p_{3}=\text { Const }
$$

The stationary condition of the Hamiltonian implies that

$$
p_{1} \cos \gamma / \omega-p_{2} \sin \gamma=(n-1) p_{3}(1-\Lambda)
$$

where

$$
\Lambda=\lambda_{\mathrm{opt}}^{n}
$$

By differentiating (23) and using the relations (11), (19), (22), we have

$$
p_{1} \sin \gamma / \omega+p_{2} \cos \gamma=(n-1) p_{3}(d A / d \gamma)
$$

Repeating the process, we have

$$
(n-1) p_{3}\left(d^{2} \Lambda / d \gamma^{2}\right)+\left(p_{2} \sin \gamma-p_{1} \cos \gamma / \omega\right)-p_{1} /\left(\omega^{2} \Lambda^{1 / n}\right)=0
$$

Equations (23) and (25) can be solved for $\omega$ and $p_{2}$ to give

$$
\begin{aligned}
\epsilon / \omega & =(n-1)[\sin \gamma(d \Lambda / d \gamma)+(1-\Lambda) \cos \gamma] \\
p_{2} & =(n-1) p_{3}[\cos \gamma(d \Lambda / d \gamma)-(1-\Lambda) \sin \gamma]
\end{aligned}
$$

where the constant $\epsilon$ is defined by

$$
\epsilon=p_{1} / p_{3}
$$

Finally, substitution of Eqs. (27)-(28) into Eq. (26) yields the following second-order nonlinear differential equation for the optimal control:

$$
\epsilon\left[d^{2} \Lambda / d \gamma^{2}+\Lambda-1\right]=\left[(n-1) / \Lambda^{1 / n}\right][\sin \gamma(d \Lambda / d \gamma)+(1-\Lambda) \cos \gamma]^{2}
$$

\section{Maximum Final Velocity with Unconstrained Range}

One integrable case of Eq. (30) is obtained when the range is free. We have $p_{1}=\epsilon=0$, and the optimal control is given by

$$
\lambda_{\mathrm{opt}}^{n}=1+a \sin \gamma
$$


where $a$ is a constant of integration to be determined by satisfying either the altitude or velocity constraint. For the maximum final velocity problem, we have the end conditions

$$
\begin{array}{lll}
\gamma=\gamma_{0}, & \xi=0, & \omega=\omega_{0}, \quad u=u_{0} \\
\gamma=\gamma_{1}, & \omega=\omega_{1}, & u \equiv \max
\end{array}
$$

The constant of integration $a$ is calculated from the end conditions in $\omega$. By integrating (11), we have

$$
\Delta \omega=\omega_{1}-\omega_{0}=-\int_{\gamma_{0}}^{\gamma_{1}} \frac{\sin \gamma}{(1+a \sin \gamma)^{1 / n}} d \gamma
$$

Here, we have a choice of a $(+)$ or $(-)$ sign in front of the radical. For positive lift, the trajectory is concave upward and, by Eq. (8), $\gamma$ is increasing. For negative lift, the trajectory is concave downward and $\gamma$ is decreasing. If $\lambda$ passes through zero and changes sign, the trajectory passes through an inflection point; hence, for the flight path angle $\gamma_{*}$ at the inflection point, we have

$$
\sin \gamma_{*}=-1 / a
$$

Equation (33) is transcendental. When $n=2$, the integral is elliptic. For other selected values of the lift exponent, namely, $n=4 / 3, n=3 / 2$, $n=5 / 2, n=3$, the integral is hyperelliptic. Because of the heat and deceleration constraints, practical maneuvers are flown at small path angles. Then, Eq. (33) is approximated by the relation

$$
\begin{aligned}
(n-1)(2-1 / n) a^{2} \Delta \omega= & \pm\left[n-(n-1) a \gamma_{1}\right]\left(1+a \gamma_{1}\right)^{1-1 / n} \\
& -\left[n-(n-1) a \gamma_{0}\right]\left(1+a \gamma_{0}\right)^{1-1 / n}
\end{aligned}
$$

where the $(+)$ sign corresponds to the case of trajectory without an inflection point. When the trajectory has an inflection point, we take the (-) sign.

In the special case $n=2$, we have the following single quartic to calculate the constant $a$ in both cases:

$$
\begin{gathered}
(81 / 16) \Delta \omega^{4} a^{4}-(9 / 2) \Delta \omega^{2}\left(\gamma_{1}^{3}+\gamma_{0}^{3}\right) a^{3}+\left[(27 / 2) \Delta \omega^{2}\left(\gamma_{1}^{2}+\gamma_{0}^{2}\right)+\left(\gamma_{1}^{3}-\gamma_{0}^{3}\right)^{2}\right] a^{2} \\
-6\left(\gamma_{1}^{2}-\gamma_{0}^{2}\right)\left(\gamma_{1}^{3}-\gamma_{0}^{3}\right) a-36 \Delta \omega^{2}+9\left(\gamma_{1}^{2}-\gamma_{0}\right)^{2}=0
\end{gathered}
$$

For $n>1$, when $a=0$, the maneuver is performed at constant angle of attack giving the maximum lift-to-drag ratio. No inflection point occurs in this case, and the end conditions satisfy the relation

$$
\omega_{1}-\cos \gamma_{1}=\omega_{0}-\cos \gamma_{0}
$$






Fig. 2. Optimum trajectories for maximum $V_{1}$.

Once the optimal lift control is determined, the range distribution is obtained by integrating Eq. (10) and the velocity distribution is obtained by integrating Eq. (12).

Figure 2 shows the optimal trajectories in the $\left(-\Delta \omega, \gamma_{1}\right)$ space with a negative initial path angle $\gamma_{0}=-10^{\circ}$ and a final path angle $\gamma_{1} \geqslant \gamma_{0}$. The curves are obtained by taking $n=2$ and using $a$ as a parameter in Eq. (35). The results check accurately with an independent computation using tabulated elliptic integrals. The admissible space is divided into the regions (A) and (B) by the composite curve $I J K$. In the region (A), the optimum path is flown without an inflection point. Points in the region (B) are reached with an inflection point on the optimal trajectory. The inflection points are on the curve $J K$, whose equation is given below.

For a terminal state along the curve $I J$, there exist two optimal trajectories, with two different lift controls, giving the same maximum final velocity. One trajectory is flown with a high lift coefficient and without an inflection point. This trajectory generates high drag, but it brings the vehicle more rapidly and in a shorter range to the terminal condition. A trajectory of this type easily violates condition (5) on the bounded lift control and is analyzed in Section 5. The second trajectory is flown with a lower lift coefficient and with an inflection point. This results in an increase in the range. This existence of two distinct classes of optimal flight paths was 


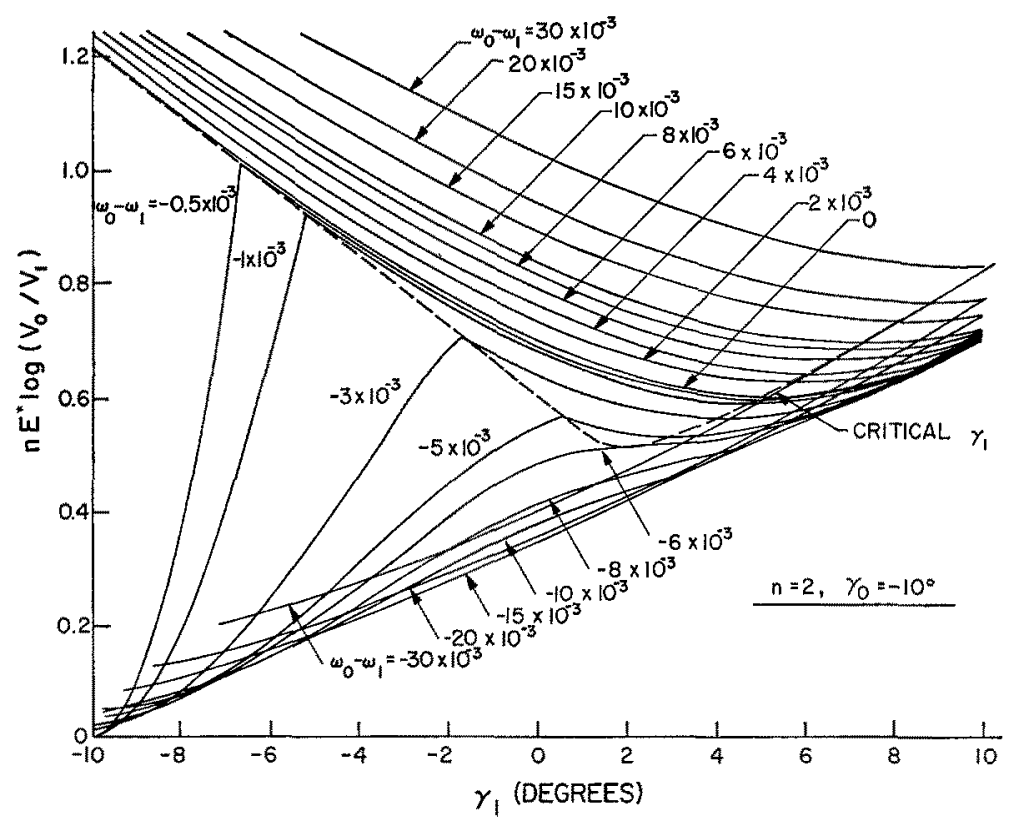

Fig. 3. Optimum final velocity loss versus $\gamma_{1}$.

detected through previous numerical investigations by Speyer, Mehra, and Bryson (Ref. 5).

Figure 3 shows the optimum velocity ratio $\alpha=n E^{*} \log \left(V_{0} / V_{1}\right)$ versus the final path angle for $n=2, \gamma_{0}=-10^{\circ}$, and different values of $\Delta \omega$.

When $\Delta \omega \leqslant 0$, i.e., when the final altitude is higher than or equal to the initial altitude, there exists one value of the exit angle $\gamma_{1}$ such that the optimum velocity loss is minimum. This value of $\gamma_{1}$ is given by

$$
\frac{d \alpha}{d \gamma_{1}}=\frac{d}{d \gamma_{1}}\left[\int_{\gamma_{0}}^{\gamma_{1}} \frac{n+a \sin \gamma}{(1+a \sin \gamma)^{1 / n}} d \gamma\right]=0
$$

or, if (33) is used, by

$$
\frac{d a}{d \gamma_{1}}\left[\Delta \omega+\int_{\gamma_{0}}^{\gamma_{1}} \frac{\sin \gamma}{(1+a \sin \gamma)^{1+1 / n}} d \gamma\right]=\frac{n}{\left(1+a \sin \gamma_{1}\right)^{1 / n}}
$$

By differentiating Eq. (33) with respect to $\gamma_{1}$, we obtain the relation

$$
\frac{d a}{d \gamma_{1}} \int_{\gamma_{0}}^{\gamma_{1}} \frac{\sin ^{2} \gamma}{n(1+a \sin \gamma)^{1+1 / n}} d \gamma=\frac{\sin \gamma_{1}}{\left(1+a \sin \gamma_{1}\right)^{1 / n}}
$$


By eliminating $\Delta \omega$ and $d a / d \gamma_{1}$ from the two equations above and Eq. (33), we have

$$
\left(1+a \sin \gamma_{1}\right) \int_{\gamma_{0}}^{\gamma_{1}} \frac{\sin ^{2} \gamma}{(1+a \sin \gamma)^{1+1 / n}} d \gamma=0
$$

Therefore,

$$
\sin \gamma_{1}=-1 / a=\sin \gamma_{*}
$$

Hence, the optimum exit angle which gives the overall minimum velocity loss for each $\Delta \omega \leqslant 0$ is the value of $\gamma_{1}$ such that the inflection point appears at the terminal position. This critical $\gamma_{1}$ is obtained along the curve $J K$ in Fig. 2. Its equation in the $\left(-\Delta \omega, \gamma_{1}\right)$ space is

$$
-\Delta \omega=\int_{\gamma_{0}}^{\gamma_{1}} \frac{\sin \gamma}{\left(1-\sin \gamma / \sin \gamma_{1}\right)^{1 / n}} d \gamma
$$

Using the small-angle approximation, we have the equation of the curve

$$
(n-1)(2-1 / n) \Delta \omega=-\gamma_{1}\left[n \gamma_{1}+(n-1) \gamma_{0}\right]\left(1-\gamma_{0} / \gamma_{1}\right)^{1-1 / n}
$$

which is a quartic for $n=2$.

When $\Delta \omega>0$, i.e., when the final altitude is lower than the initial altitude, both a relative maximum velocity loss and a relative minimum velocity loss exist. The maximum occurs when we switch from optimum flight without an inflection point to optimum flight with an inflection point. For each $\Delta \omega$, the corresponding value of $\gamma_{1}$ is given by the curve $I J$ in Fig. 2 . The value of $\gamma_{1}$ for relative minimum velocity loss is given by Eq. (41).

When

$$
\Delta \omega>\frac{[\sqrt{ }(2 n-1)-1]^{2} \gamma_{0}^{2}}{4(n-1) \sqrt{ }(2 n-1)}\left[\frac{2 n-1+n \sqrt[V]{ }(2 n-1)}{n-1}\right]^{1-1 / n}
$$

the relative minimum ceases to exist and all the optimal trajectories are flown without an inflection point. If we also consider trajectories for which $\gamma_{1}<\gamma_{0}$, then, for each $\Delta \omega>0$, there is always a critical $\gamma_{1}<\gamma_{0}$, given by Eq. (42), such that the overall velocity loss is a minimum.

We also note that, for each final angle $\gamma_{1}$, a value of $\Delta \omega$ exists such that the velocity loss is a minimum. The trajectory is flown at maximum aerodynamic efficiency $(a=0)$ and $\Delta \omega$ is given by Eq. (37). The line $\alpha=n\left(\gamma_{1}-\gamma_{0}\right)$ is the envelope of the curves in Fig. 3.

The trajectories presented in this section are optimal in the sense that they give the minimum velocity loss. Some trajectories require high positive 
lift, and this is not admissible if the lift control is bounded. In these cases, bounded control can be optimal. Discussion of these trajectories is the subject of Section 5.

\section{Maximum Final Altitude with Unconstrained Range}

In this case, the range is free and the optimal control is given by Eq. (31). We assume that the terminal flight path angle $\gamma_{1}$ and the velocity loss $\alpha$ are prescribed, and we wish to find the trajectory which maximizes the final altitude. The end conditions are

$$
\begin{array}{ll}
\gamma=\gamma_{0}, & \xi=0, \quad u=u_{0}, \quad \omega=\omega_{0} \\
\gamma=\gamma_{1}, & u=u_{1}, \quad-\omega_{1} \equiv \max
\end{array}
$$

The results are similar to those obtained in the case of maximum final velocity. First, the integration constant $a$ is calculated from the end conditions in $u$. By integrating (12), we have

$$
\alpha=n E^{*} \log \left(V_{0} / V_{1}\right)=\int_{\gamma_{0}}^{\gamma_{1}} \frac{n+a \sin \gamma}{(1+a \sin \gamma)^{1 / n}} d \gamma
$$

For small path angles, we have the approximate equation

$$
(2-1 / n) a \alpha= \pm\left(2 n+a \gamma_{1}\right)\left(1+a \gamma_{1}\right)^{1-1 / n}-\left(2 n+a \gamma_{0}\right)\left(1+a \gamma_{0}\right)^{1-1 / n}
$$

where the $(+)$ sign corresponds to the case of a trajectory without an inflection point. When the trajectory has an inflection point we take the $(-)$ sign. In the special case $n=2$, we have the following single quartic to calculate the constant $a$ in both cases:

$$
\begin{aligned}
& \left(\gamma_{1}^{3}-\gamma_{0}^{3}\right)^{2} a^{4}-9\left[(1 / 2)\left(\gamma_{1}^{3}+\gamma_{0}^{3}\right) \delta-4 \gamma_{1} \gamma_{0}\left(\gamma_{1}+\gamma_{0}\right)\left(\gamma_{1}-\gamma_{0}\right)^{2}\right] a^{3} \\
& \quad+3\left[(27 / 16) \delta^{2}-27 \gamma_{1} \gamma_{0} \delta+16\left(\gamma_{1}-\gamma_{0}\right)\left(\gamma_{1}^{3}-\gamma_{0}^{3}\right)\right] a^{2} \\
& \quad-108\left(\gamma_{1}+\gamma_{0}\right) \delta a-144 \delta=0
\end{aligned}
$$

where

$$
\delta=\alpha^{2}-4\left(\gamma_{1}-\gamma_{0}\right)^{2}
$$

For any $n>1$, the optimum path is flown at constant angle of attack giving maximum lift-to-drag ratio when the velocity loss is such that

$$
V_{0} / V_{1}=\exp \left[\left(\gamma_{1}-\gamma_{0}\right) / E\right]
$$




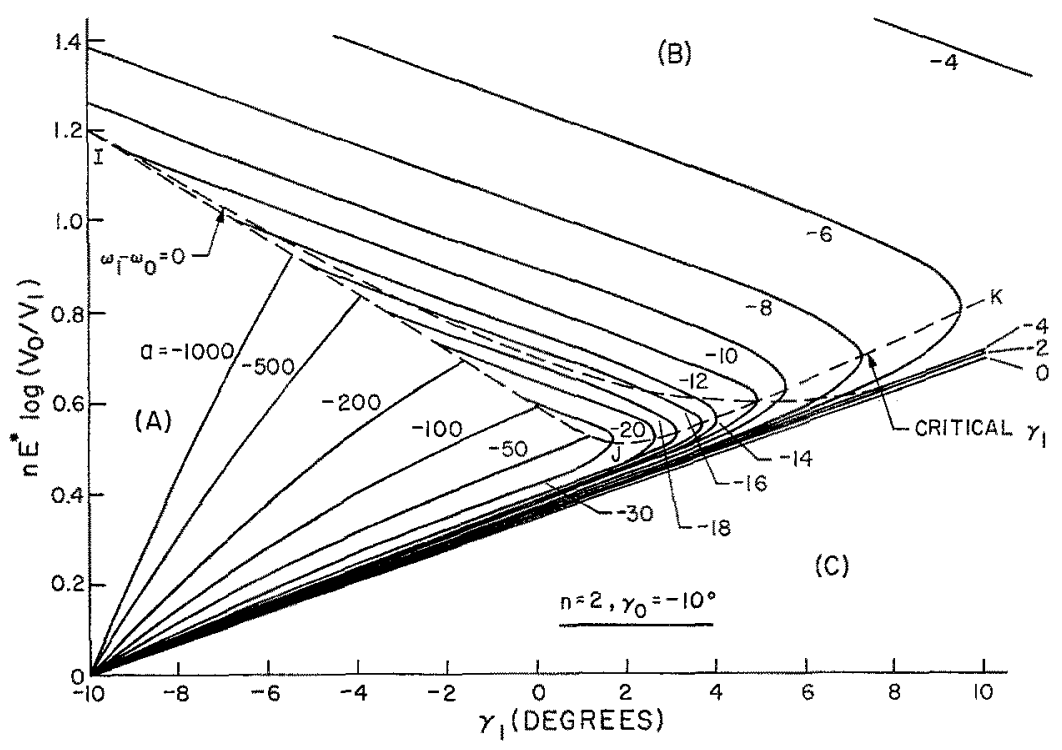

Fig. 4. Optimum trajectories for maximum final altitude.

Once the optimal lift control is determined, the range distribution is obtained by integrating Eq. (10) and the altitude distribution is obtained by integrating Eq. (11).

Figure 4 shows the optimal trajectories in the $\left(\alpha, \gamma_{1}\right)$ space with a negative initial path angle $\gamma_{0}=-10^{\circ}$ and a terminal path angle $\gamma_{1} \geqslant \gamma_{0}$. The curves are obtained by taking $n=2$ and using $a$ as a parameter in the approximate equation (46). The admissible space is divided into the regions (A) and (B) by the composite curve $I J K$. In the region (A), optimal trajectories are flown without an inflection point. Points in the region (B) can be reached by optimal trajectories with an inflection point. The inflection point occurs along the curve $J K$, whose equation is given below. For a terminal state along the curve $I J$, there exist two optimal trajectories, with different lift controls, giving the same maximum final altitude. One trajectory is flown with high lift and without an inflection point. This trajectory easily violates condition (5) on bounded lift control. The other trajectory requires lower lift and has an inflection point on it. In the graph, we notice a region (C) that no trajectory can reach. This region is below the line $a=0$, that is, below the line $\alpha=n\left(\gamma_{1}-\gamma_{0}\right)$. Below this line, the prescribed velocity loss is too small for the vehicle to reach the given final path angle. This is the problem of optimum rotation from a given $\gamma_{0}$ to a given $\gamma_{1}$ with the least velocity loss while the 


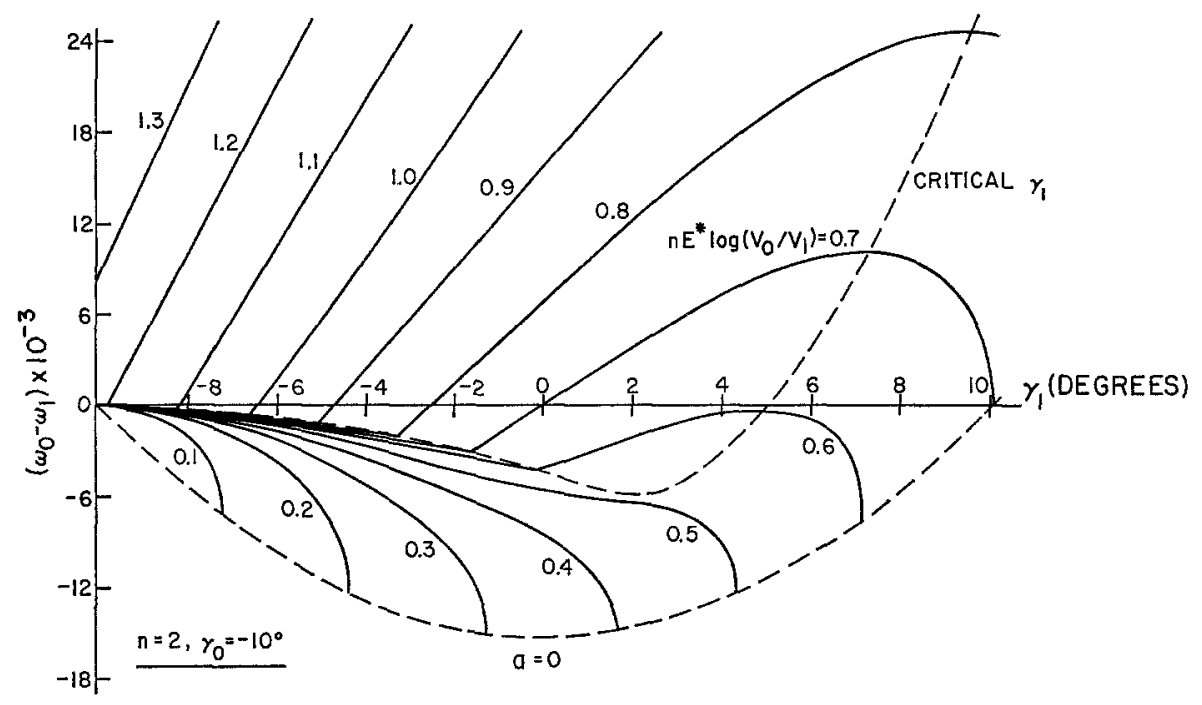

Fig. 5. Optimum altitude gain for available velocity loss versus $\gamma_{1}$.

final position is not prescribed. Hence, it is a subclass of the problem treated in the preceding section.

Figure 5 shows the altitude gain $-\Delta \omega$ versus the terminal path angle $\gamma_{1}$ for $n=2, \gamma_{0}=-10^{\circ}$, and different values of the velocity loss. For a given velocity loss, a maximum attainable final angle exists and is given by

$$
\gamma_{1}=\alpha / n+\gamma_{0}
$$

When the given velocity loss is large enough, a terminal path angle $\gamma_{1}$ exists such that the optimum altitude gain is a maximum. This angle is such that the inflection point appears at the terminal position. It is obtained along the curve $J K$ in Fig. 4 , and its equation in the $\left(\alpha, \gamma_{1}\right)$ space is

$$
\alpha=\int_{\nu_{0}}^{\gamma_{1}} \frac{n-\sin \gamma / \sin \gamma_{1}}{\left[1-\sin \gamma / \sin \gamma_{1}\right]^{1 / n}} d \gamma
$$

Using the small-angle approximation, we have the equation of the curve

$$
(2-1 / n) \alpha=\left(2 n \gamma_{1}-\gamma_{0}\right)\left(1-\gamma_{0} / \gamma_{1}\right)^{1-1 / n}
$$

which is a cubic for $n=2$. When

$$
\alpha<-n[(2 n-1) /(n-1)]^{1-1 / n} \gamma_{0}
$$


the maximum is only relative, since the final altitude is always below the initial altitude. When

$$
\alpha<-\frac{n \gamma_{0}}{\sqrt{ }(2 n-1)}\left[\frac{2 n-1+n \sqrt{ }(2 n-1)}{n-1}\right]^{1-1 / n}
$$

the relative maximum altitude gain ceases to exist.

\section{Optimal Trajectories with Bounded Lift Control}

A simple check in Figs. 2 and 4 shows that, for certain prescribed terminal states, the constant $a$ is such that $\lambda_{\text {opt }}$ can become very large; hence, if condition (5) on the bounded control is enforced, the optimal trajectory is not admissible. In this section, we study the case where bounded control is optimal. For definiteness, we consider a negative initial path angle. If an inflection point exists, the maximum angle $\gamma_{*}$ is such that

$$
-\pi / 2<\gamma_{0} \leqslant \gamma_{1} \leqslant \gamma_{*}<\pi / 2
$$

First, we consider the case of maximum final velocity; next, we consider the case of maximum final altitude.

5.1. Maximum Final Velocity, Since the range is free and the final velocity is to be maximized,

$$
p_{1}=0, \quad p_{2}=\text { Const }=-(n-1) a, \quad p_{3}=-1
$$

The control is given by

$$
\begin{aligned}
& \lambda^{n}=1+a \sin \gamma \\
& \lambda^{n} \leqslant \lambda_{\max }^{n}
\end{aligned}
$$

The Hamiltonian (17) takes the form

$$
H=(1 / \gamma)\left[(n-1)(1+a \sin \gamma)+\lambda^{n}\right]
$$

Initially, $\lambda$ is positive; if an inflection point exists, $\lambda$ changes sign beyond the inflection point. Since the variable $\gamma$ decreases beyond the inflection point, we should change the sign of the right-hand side of relation (59). Hence, expression (59) is always valid if we take the positive sign for $\lambda$. 
Figure 6 shows the variation of $H$ with respect to $\lambda$. The curve has an absolute minimum at

$$
\lambda=(1+a \sin \gamma)^{1 / n}
$$

and is asymptotic to the curve $H=\lambda^{n-1}$ for $n>1, \lambda \rightarrow \infty$. It is clear that, if

$$
(1+a \sin \gamma)^{1 / n}>\lambda_{\max }
$$

then $\lambda_{\max }$ is the control satisfying (58) while giving the minimum of $H$. Hence, $\lambda_{\max }$ is the optimal control in this case.

Figure 7 shows the variation of $\lambda^{n}$, given by (57), with respect to $\gamma$ for $a>0$. The curve intersects the line $\lambda^{n}=\lambda_{\max }^{n}$ at, at most, one point between $\lambda_{0}$ and $\lambda_{1}$. There is no inflection point in this case. Hence, if bounded control is encountered, the sequence is

$$
\text { Variable control } \rightarrow \text { Bounded control }
$$

To calculate the constant $a$ for variable control and the path angle $\gamma_{s}$ at which we switch control, we first note that $\gamma_{s}$ is such that

$$
\lambda_{\max }^{n}=1+a \sin \gamma_{s}
$$

Next, if we integrate Eq. (11) from $\gamma_{0}$ to $\gamma_{s}$ with variable control (57), we have an equation of the form

$$
\omega_{s}-\omega_{0}=f_{1}\left(\gamma_{0}, \gamma_{s}, a\right)
$$

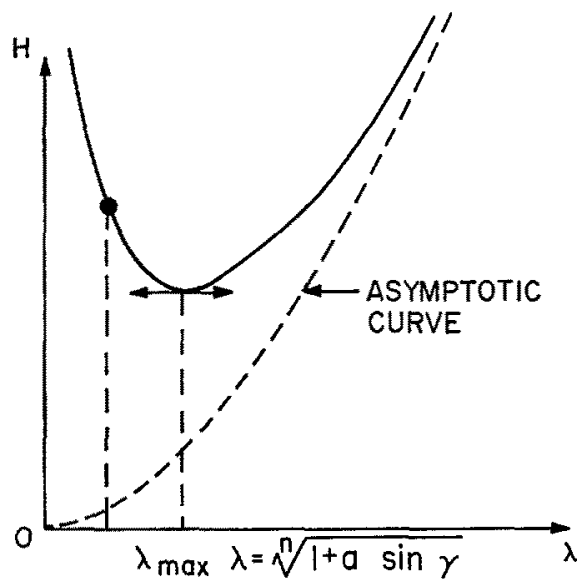

Fig. 6. Variation of the Hamiltonian with respect to $\lambda$. 


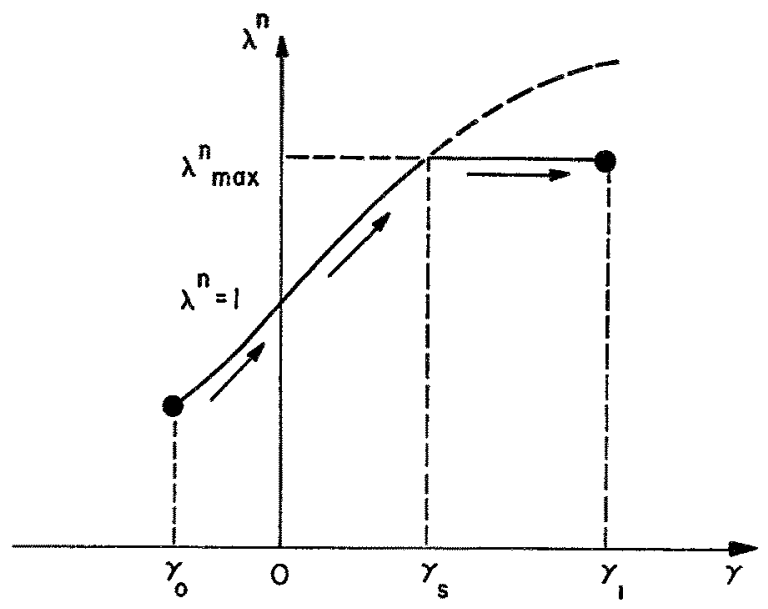

Fig. 7. Variation of $\lambda_{\text {opt }}^{n}(a>0)$.

where $\omega_{s}$ is the altitude corresponding to $\gamma_{s}$, that is, the switching altitude. Finally, if (11) is integrated from $\gamma_{s}$ to $\gamma_{1}$ with $\lambda=\lambda_{\max }$, we have

$$
\omega_{1}-\omega_{s}=\left(\cos \gamma_{1}-\cos \gamma_{s}\right) / \lambda_{\max }
$$

Equations (61)-(63) permit the calculation of the constant $a$, the switching angle $\gamma_{s}$, and the switching altitude $\omega_{s}$.

If the small-angle approximation is used, the equations are replaced by

$$
\begin{aligned}
\lambda_{\max }^{n}= & 1+a \gamma_{s} \\
\omega_{s}-\omega_{0}= & {\left[n /(n-1)(2 n-1) a^{2}\right]\left\{\left[n-(n-1) a \gamma_{s}\right]\left(1+a \gamma_{s}\right)^{1-1 / n}\right.} \\
& \left.-\left[n-(n-1) a \gamma_{0}\right]\left(1+a \gamma_{0}\right)^{1-1 / n}\right\} \\
\omega_{1}-\omega_{s}= & \left(\gamma_{s}^{2}-\gamma_{1}^{2}\right) / 2 \lambda_{\max }
\end{aligned}
$$

For $n=2$, the resulting equation is a quartic in $a$.

Figure 8 shows the variation of $\lambda^{n}$, given by (57), with respect to $\gamma$ for $a<0$. The curve intersects the line $\lambda^{n}=\lambda_{\max }^{n}$ at, at most, one point. An inflection point may exist in this case, since $\lambda$ may vanish. If $\gamma_{1}>\gamma_{s}$, the optimal control sequence is

$$
\text { Bounded control } \rightarrow \text { Variable control }
$$






Fig. 8. Variation of $\lambda_{\mathrm{opt}}^{n}(a<0)$.

Variable control may be flown with or without an inflection point. To calculate the constant $a$, the switching angle $\gamma_{s}$, and the switching altitude $\omega_{s}$, we first integrate Eq. (11) from $\gamma_{0}$ to $\gamma_{s}$ with $\lambda=\lambda_{\max }$ and next from $\gamma_{s}$ to $\gamma_{1}$ with the variable control (57). For small angles, we have the system

$$
\begin{aligned}
\lambda_{\max }^{n}= & 1+a \gamma_{s} \\
\omega_{s}-\omega_{0}= & \left(\gamma_{0}^{2}-\gamma_{s}^{2}\right) / 2 \lambda_{\max } \\
\omega_{1}-\omega_{s}= & {\left[n /(n-1)(2 n-1) a^{2}\right]\left\{ \pm\left[n-(n-1) a \gamma_{1}\right]\left(1+a \gamma_{1}\right)^{1-1 / n}\right.} \\
& \left.-\left[n-(n-1) a \gamma_{s}\right]\left(1+a \gamma_{s}\right)^{1-1 / n}\right\}
\end{aligned}
$$

In the last equation, the $(+)$ sign corresponds to the case of a trajectory without an inflection point. For $n=2$, the resulting equation of the system is a quartic in $a$.

It is possible that, for the case $a<0$, the end conditions and the value of $\lambda_{\max }$ are such that $\gamma_{1}<\gamma_{s}$ (Fig. 9). For this case, it is necessary that an inflection point exists, as can be seen in the figure; the optimal control sequence is

Bounded control $\rightarrow$ Variable control with an inflection point $\rightarrow$ Bounded control

To calculate the optimal elements involved, we integrate Eq. (11) using for $\lambda$ the optimal sequence (67). The lift is positive before the inflection point and is negative afterward. 


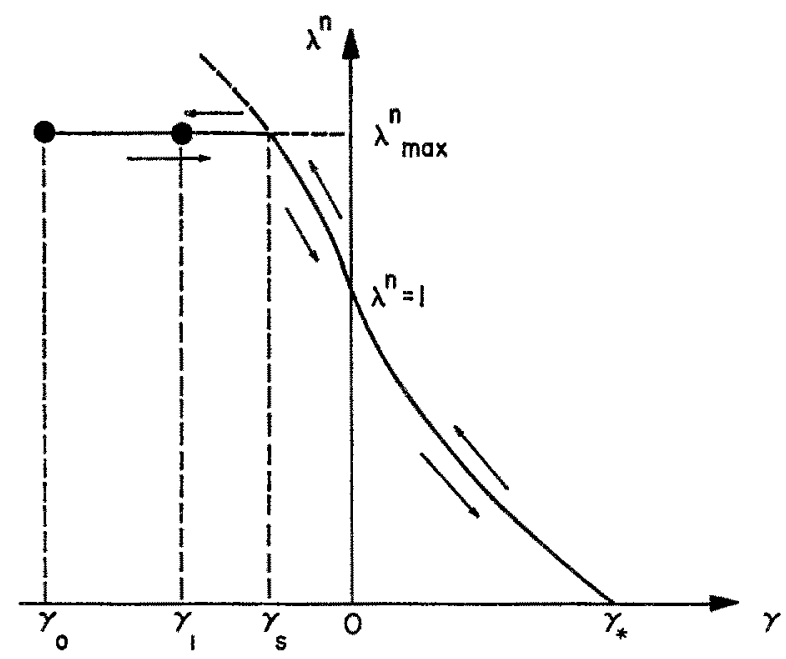

Fig. 9. Case of two switchings.

For small angles, we have the system

$$
\begin{aligned}
\lambda_{\max }^{n} & =1+a \gamma_{s} \\
\omega_{s 1}-\omega_{0} & =\left(\gamma_{0}^{2}-\gamma_{s}^{2}\right) / 2 \lambda_{\max } \\
\omega_{s 2}-\omega_{s 1} & =-\left[2 n /(n-1)(2 n-1) a^{2}\right]\left[n-(n-1) a \gamma_{s}\right]\left(1+a \gamma_{s}\right)^{1-1 / n} \\
\omega_{1}-\omega_{s 2} & =\left(\gamma_{1}^{2}-\gamma_{s}^{2}\right) / 2 \lambda_{\max }
\end{aligned}
$$

where $\omega_{s 1}$ and $\omega_{s 2}$ are, respectively, the first switching altitude and the second switching altitude (Fig. 10).

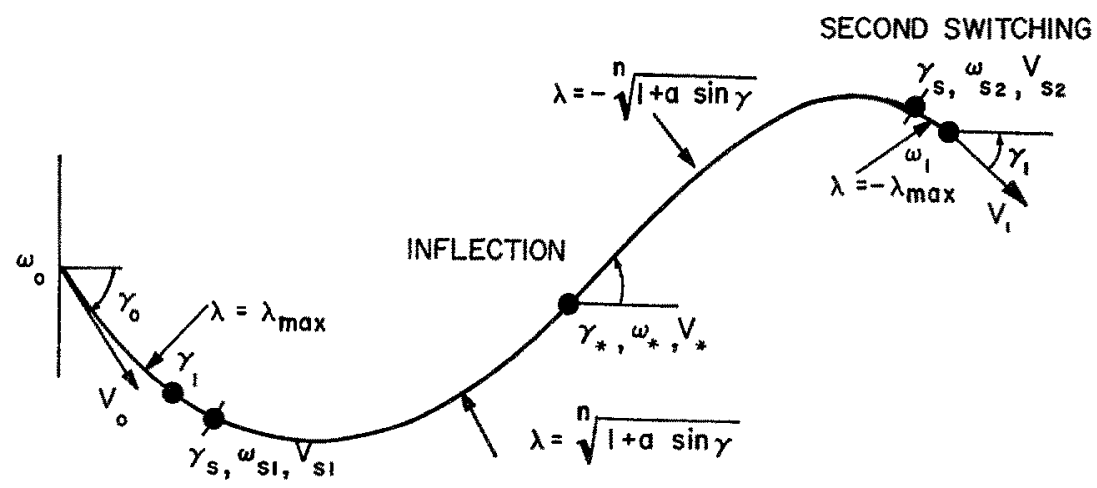

FIRST SWITCHING

Fig. 10. Optimal flight path with two switchings. 
The system can be solved to give

$$
a=-\sqrt{\left[2 \frac{(1-n) \lambda_{\max }^{2 n}+2(2 n-1) \lambda_{\max }^{n}+(n-1)(2 n-1)}{(n-1)(2 n-1)\left(\gamma_{0}^{2}+\gamma_{1}^{2}-2 \lambda \Delta \omega\right)}\right]}
$$

From this value of $a$, we can calculate $\gamma_{s}, \omega_{s 1}, \omega_{s 2}$. If

$$
\lambda_{\max }^{n}<[2 n-1+n \sqrt{ }(2 n-1)] /(n-1)
$$

we must have

$$
\Delta \omega<\left(\gamma_{0}^{2}+\gamma_{1}^{2}\right) / 2 \lambda_{\max }
$$

Otherwise, both inequalities reverse for real values of $a$.

Since bounded control interferes only with high-lift trajectories, it is possible for the same end condition to find a lower-lift variety with a smaller velocity loss, as explained before. Hence, for the case of bounded control, we must compare the trajectory with an alternate lower-lift trajectory to find the true optimal trajectory.

5.2. Maximum Final Altitude. For this case, the arguments are the same as for the case of maximum final velocity. The integration of Eq. (11) is to be replaced by the integration of Eq. (12). Also, we note that only negative values of the constant $a$ lead to a maximum final altitude. We give here the relations to calculate the optimal elements for the case of small path angles.

In the case of one switching, we have the system

$$
\begin{aligned}
\lambda_{\max }^{n}= & 1+a \gamma_{s} \\
n E^{*} \log \left(V_{0} / V_{s}\right)= & {\left[n-1+\lambda_{\max }^{n}\right]\left(\gamma_{s}-\gamma_{0}\right) / \lambda_{\max } } \\
n E^{*} \log \left(V_{s} / V_{1}\right)= & {[n /(2 n-1) a]\left[ \pm\left(2 n+a \gamma_{1}\right)\left(1+a \gamma_{1}\right)^{1-1 / n}\right.} \\
& \left.-\left(2 n+a \gamma_{s}\right)\left(1+a \gamma_{s}\right)^{1-1 / n}\right]
\end{aligned}
$$

where $V_{s}$ is the velocity at the switching point. In the last equation, the $(+)$ sign corresponds to the case of trajectory without an inflection point. For $n=2$, the resulting equation is a cubic in $a$.

In the case of two switchings, the variable-lift portion of the trajectory is flown with an inflection point and the solution is obtained from the system

$$
\begin{aligned}
\lambda_{\text {max }}^{n} & =1+a \gamma_{s} \\
n E^{*} \log \left(V_{0} / V_{s 1}\right) & =\left[n-1+\lambda_{\max }^{n}\right]\left(\gamma_{s}-\gamma_{0}\right) / \lambda_{\max } \\
n E^{*} \log \left(V_{s 1} / V_{s 2}\right) & =[2 n /(2 n-1) a]\left(2 n+a \gamma_{s}\right)\left(1+a \gamma_{s}\right)^{1-1 / n} \\
n E^{*} \log \left(V_{s 2 /} / V_{1}\right) & =\left[n-1+\lambda_{\max }^{n}\right]\left(\gamma_{s}-\gamma_{1}\right) / \lambda_{\max }
\end{aligned}
$$


where $V_{s 1}$ and $V_{s 2}$ are, respectively, the first switching velocity and the second switching velocity (Fig. 10). The system can be solved to give

$$
a=\frac{2}{2 n-1} \frac{(n-1) \lambda_{\max }^{2 n}-2(2 n-1) \lambda_{\max }^{n}-(n-1)(2 n-1)}{\left[n-1+\lambda_{\max }^{n}\right]\left(\gamma_{0}+\gamma_{1}\right)+\lambda_{\max }{ }^{\alpha}}
$$

From this value of $a$, we can calculate $\gamma_{s}, V_{s 1}, V_{s 2}$. If

$$
\lambda_{\max }^{n}<[2 n-1+n \sqrt{ }(2 n-1)] /(n-1)
$$

we must have

$$
\alpha>-\left[n-1+\lambda_{\max }^{n}\right]\left(\gamma_{0}+\gamma_{1}\right) / \lambda_{\max }
$$

Otherwise, both inequalities reverse for $a$ to have negative values.

\section{Conclusions}

In this paper, analytical solutions of some problems concerning the optimum maneuvering of a lifting vehicle in the hypervelocity regime are obtained using the approximation of Allen and Eggers. The characteristic properties of the optimal trajectories are proved in the most general form, using a generalized drag polar.

When the range is free, the optimal lift control is obtained in closed form. With this control law, a family of optimal flight paths is generated. It is seen that two distinct classes of optimal paths exist. They arise from widely different control strategies. One flight path uses high lift (and, hence, large drag) to bring the vehicle more rapidly and in a shorter range to the prescribed end conditions. The other flight path is flown with low angle-ofattack to keep the drag small, but it requires more time and range to get to the final condition.

For the maximum final velocity problem, the control law is determined completely if only the initial and terminal altitude and path angle are known. For the maximum final altitude problem, the prescribed initial and terminal velocity and path angle are used to determine the optimal control. It is shown that there exists one optimum exit angle. This angle is such that the lift vanishes at the terminal position.

The optimal flight path is flown with or without an inflection point.

The study includes the case where the lift control is bounded. In this case, bounded control is optimal whenever it is reached. The switching sequences for different cases are discussed. It is shown that, for the case 
of free range, there are, at most, two switchings. Bounded lift control is always at the ends of the optimal trajectory.

When the range is prescribed, the problem consists of integrating a second-order nonlinear differential equation.

\section{References}

1. Contensou, P., Contribution à l'Étude Schématique des Trajectoires Semi-Ballistiques à Grande Portée, Paper communicated to the "Association Technique Maritime et Aéronautique," Paris, France, 1965.

2. Allen, H. J., and EgGers, A. J., JR., A Study of the Motion and Aerodynamic Heating of Missiles Entering the Earth's Atmosphere at High Supersonic Speeds, NACA Report No. 1381, 1958.

3. Eggers, A. J., Jr., Allen, H. J., and Neice, S. E., A Comparative Analysis of the Performance of Long-Range Hypervelocity Vehicles, NACA Report No. 1382, 1958.

4. Mrele, A., Flight Mechanics, Vol. 1: Theory of Flight Paths, Addison-Wesley Publishing Company, Reading, Massachusetts, 1962.

5. Speyer, J. L., Mehra, R. K., and Bryson, A. E., JR., The Separate Computation of Arcs for Optimal Flight Paths with State Variable Inequality Constraints, Paper presented at the Colloquium on Advanced Problems and Methods for Space Flight Optimization, Liège, Belgium, 1967. 